\title{
How to select the most appropriate adjuvant treatment after neoadjuvant treatment and resection for locally advanced pancreatic cancer?
}

\author{
Elena Rangelova ${ }^{1,2}$, Svein Olav Bratlie ${ }^{1,2}$ \\ ${ }^{1}$ Section for Upper Abdominal Surgery at Department of Surgery, Sahlgrenska University Hospital, Gothenburg, Sweden; ${ }^{2}$ Department of Surgery \\ at The Institute of Clinical Sciences, Sahlgrenska Academy, University of Gothenburg, Gothenburg, Sweden \\ Contributions: (I) Conception and design: E Rangelova; (II) Administrative support: Both authors; (III) Provision of study material or patients: Both \\ authors; (IV) Collection and assembly of data: E Rangelova; (V) Data analysis and interpretation: Both authors; (VI) Manuscript writing: Both \\ authors; (VII) Final approval of manuscript: Both authors. \\ Correspondence to: Elena Rangelova, MD, PhD, FACS. Department of Upper Abdominal Surgery, Sahlgrenska University Hospital, Blå Stråket 5, \\ floor 2, 41345 Gothenburg, Sweden. Email: elena.rangelova@gu.se.
}

\begin{abstract}
Adjuvant chemotherapy (ACT) significantly improves survival of patients undergoing upfront surgery for resectable pancreatic cancer. After introducing the concept of neoadjuvant therapy (NAT) with potent chemotherapy regimens, long term survival has been achieved even in patients with borderline and locally advanced pancreatic cancer (BR/LAPC) following radical resection. The observed pathologic tumor response is strongly predictive of survival and provides a unique opportunity to visualize to what extent the cancer has been sensitive to the administered chemotherapy regimen and may potentially give hint how to personalize further oncologic treatment. Current literature provides only limited and heterogeneous data as to whether and what type of ACT is beneficial after NAT and resection for BR/LAPC. Larger studies suggest that ACT may bring survival advantage and should be attempted particularly in node-positive disease and preferably with more potent regimen such as FOLFIRINOX, if tolerable. In case of complete pathologic response, particularly after FOLFIRINOX, it does not seem beneficial to deescalate the treatment during ACT, but whether continuation on the same regimen is worthwhile needs to be further examined. In case of gemcitabine-based treatment as NAT, continuation with more cycles seems to be of value unless tumor biology proves to be too aggressive, with high lymph node ratio. Whether switch to a different regimen should be sought, if tolerability allows it, needs to be further studied. Whether it is the exact treatment sequence (NAT, ACT or both) of the potent chemotherapy regimens like FOLFIRINOX and gemcitabinenab-paclitaxel or the total dose of chemotherapy that has impact on survival in BR/LAPC, is unknown.
\end{abstract}

Keywords: Locally advanced pancreatic cancer (LAPC); borderline; resection; adjuvant therapy; neoadjuvant therapy

Submitted Aug 04, 2021. Accepted for publication Sep 22, 2021.

doi: $10.21037 /$ jgo-21-474

View this article at: https://dx.doi.org/10.21037/jgo-21-474

$\wedge$ ORCID: 0000-0003-3844-7265. 


\section{Introduction}

Surgical resection is the treatment modality that must be involved if long-term survival of pancreatic cancer (PC) is to be achieved (1). Even after resection, about $30 \%$ of patients will develop recurrence within the first year of surgery and further up to $75-85 \%$ within 5 years, with fatal outcome $(2,3)$. That implies that even smaller resectable tumors may give rise to indolent systemic spread that cannot be addressed solely by the local treatment that surgery is. The addition of adjuvant chemotherapy (ACT) after surgery, to address the problem of occult residual systemic metastatic disease, has almost cut in half the risk for recurrence and thus improved the survival of patients with resectable PC $(4,5)$. The series of ESPAC trials established gemcitabine and, later on, gemcitabine in combination with capecitabine as the standard of adjuvant therapy, particularly in Europe $(4,6)$. In Asian population, S-1 has been applied as an alternative of choice (7). As for borderline (BR) and locally advanced pancreatic cancer (LAPC), the same treatment sequence of upfront surgery followed by gemcitabine-based ACT, had not proven to give the same survival advantage (8). Thus, the skepticism towards the benefit of surgery in BR and LAPC has for long ruled the surgical opinion.

The introduction of FOLFIRINOX and gemcitabinenab-paclitaxel significantly prolonged the survival of patients with unresectable and metastatic disease $(9,10)$. Furthermore, pretreatment with these potent regimens of patients with BR and LAPC, followed by surgical resection, resulted in the observation of long-term survivors and revived the interest towards neoadjuvant treatment (NAT) (11-14). The concept of NAT in PC has been widely picked up now, irrespective of local resectability status, and multiple trials are ongoing worldwide $(15,16)$. NAT has the potential advantages of not only increasing the pool of patients receiving systemic therapy, since tolerability may be impaired after preceding major surgery, but also selecting the better surgical candidates, having more favorable tumor biology and responding to the applied oncologic therapy $(11,12,17,18)$. FOLFIRINOX has also proved to be superior to gemcitabine in the adjuvant setting after resection, with 3 -year survival of $63.4 \%$ versus $48.6 \%$, respectively, thus becoming the new adjuvant gold standard, whenever tolerable (19). The question remains whether ACT still carries the same survival benefit in the context of preceding NAT and resection. Is it the timing, the potency or the personalized choice of chemotherapy that plays the decisive role for survival?
Most of the factors predictive of prognosis of BR and LAPC are attained from the final histological assessment of the resected specimen (20-24). The observed tumor regression provides a unique opportunity to practically evaluate to which extent patients have been responsive to the given oncologic treatment and potentially could hint what drugs should be more appropriate in the postoperative setting. In case of the most common partial pathologic response, it is unclear whether the optimal drug choice should be the one that has proven effective, as reflected by the presence of destructed tumor areas, or an alternative should be sought in order to address the residual nonresponsive viable tumor. There are theoretical advantages to both scenarios. When radiotherapy comes into context, though, it becomes even more cumbersome to interpret histologic regression as guidance to further treatment. The regression grade reflects the potency of radiation itself but does not guarantee that potential systemic spread has not occurred already and hint which drug would best approach it.

The purpose of this review is to summarize the available evidence for the utility of ACT after preceding NAT for borderline and locally advanced pancreatic cancer. A systemic literature search in PubMed was performed to identify series reporting on the outcome of resected patients with pancreatic adenocarcinoma after NAT. Of these, articles reporting on series that included only BR/LAPC or where these were part of the cohorts were reviewed in detail, while series reporting solely on primary resectable PC were excluded. Of the relevant publications, only articles that reported survival outcome after ACT were assessed and summarized in Table 1 in order to attempt to answer four clinical questions-whether there is benefit of ACT after NAT, particularly for patients with BR/LAPC; is there a subgroup of patients having (larger) benefit and which type of ACT should be chosen.

\section{Is there benefit of ACT after NAT?}

The interest in NAT started to escalate exponentially recently, since 2011, after the report on the effectiveness of FOLFIRINOX in unresectable PC was published (9). Naturally, the studies investigating the usage of ACT after NAT are falling behind and data on its effectiveness has not always been reported in them (25-27). Currently, there are no randomized controlled trials assessing the utility of ACT after NAT and resection for PC. All evidence comes from retrospective studies, mostly published in 2019- 


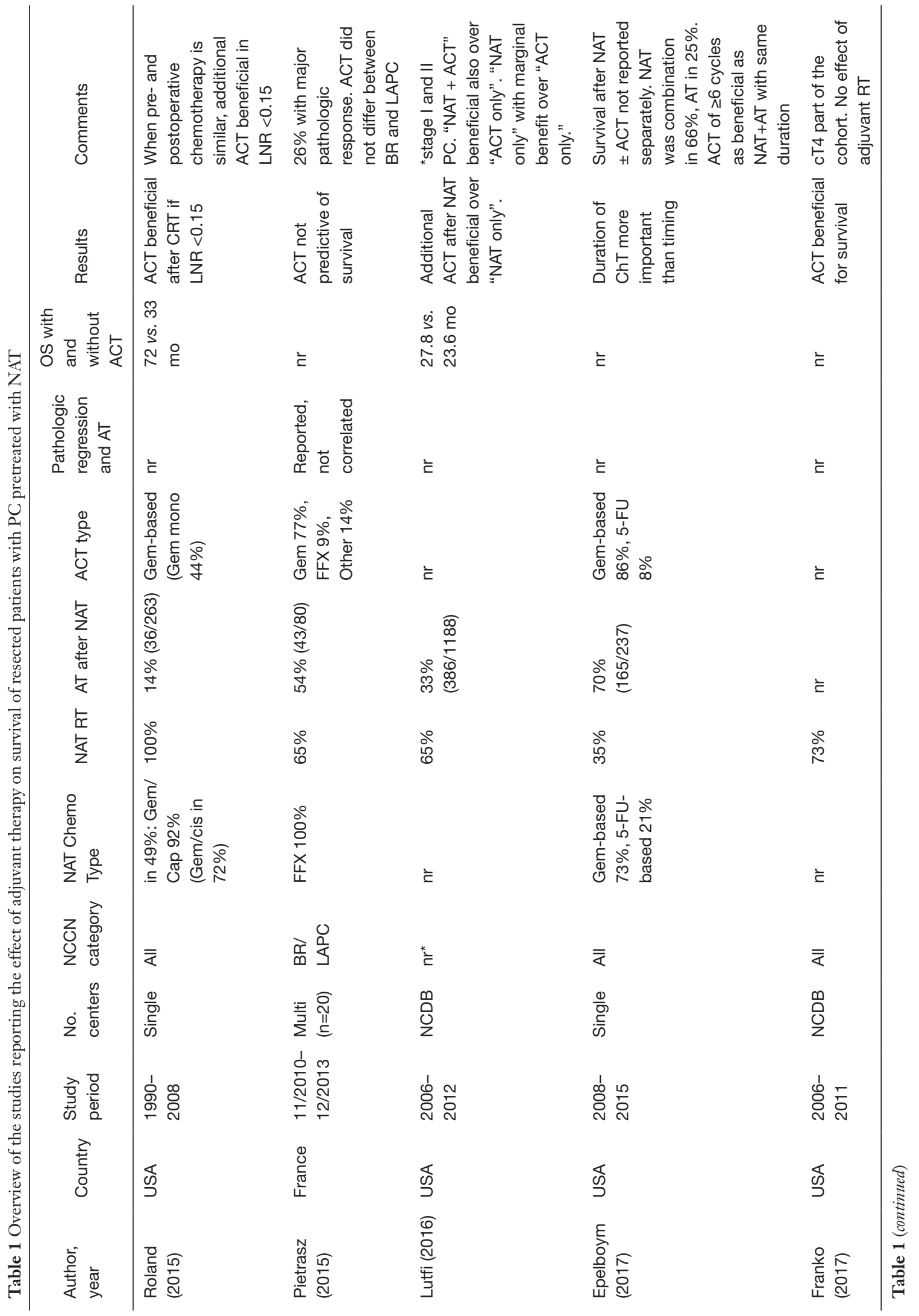




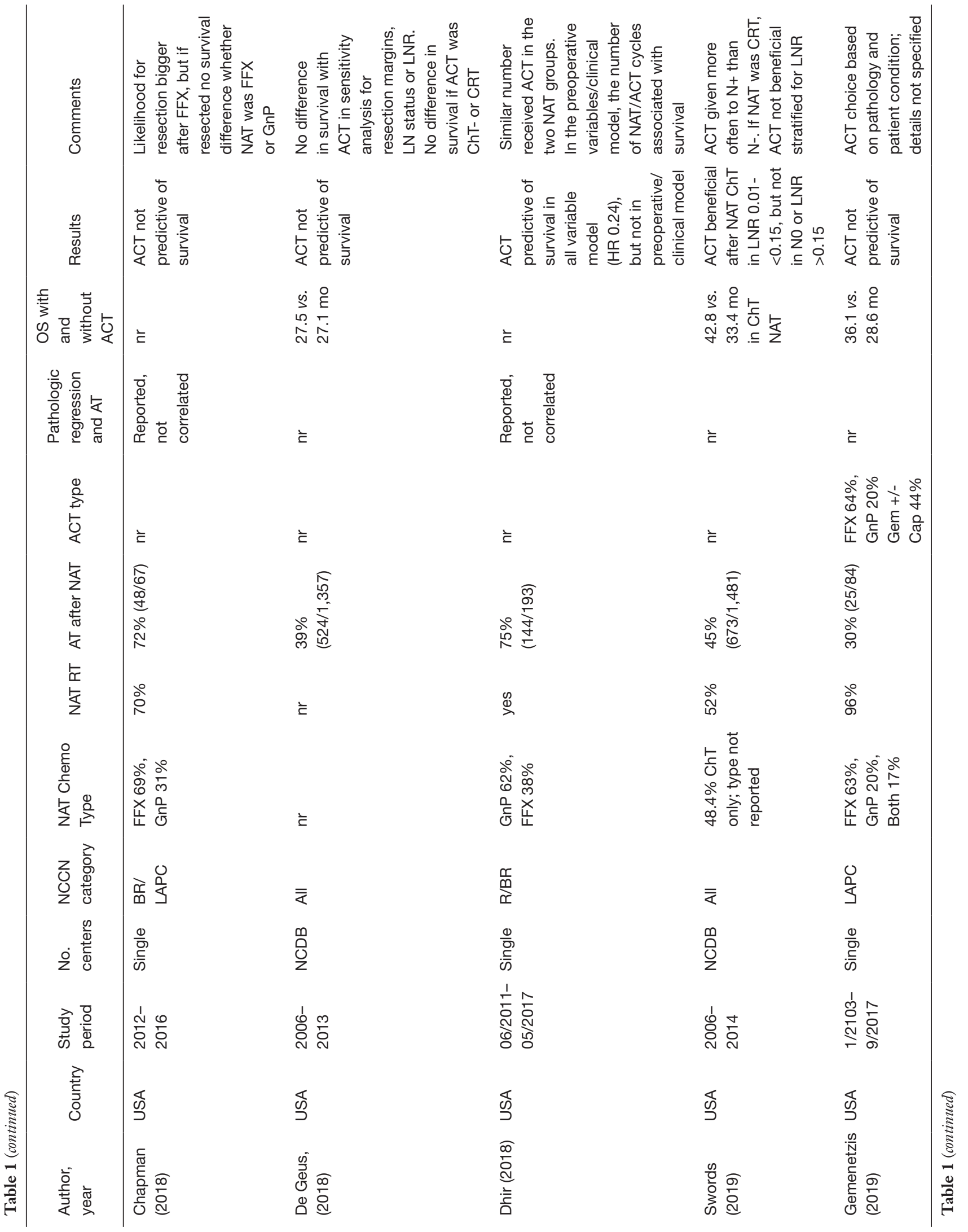




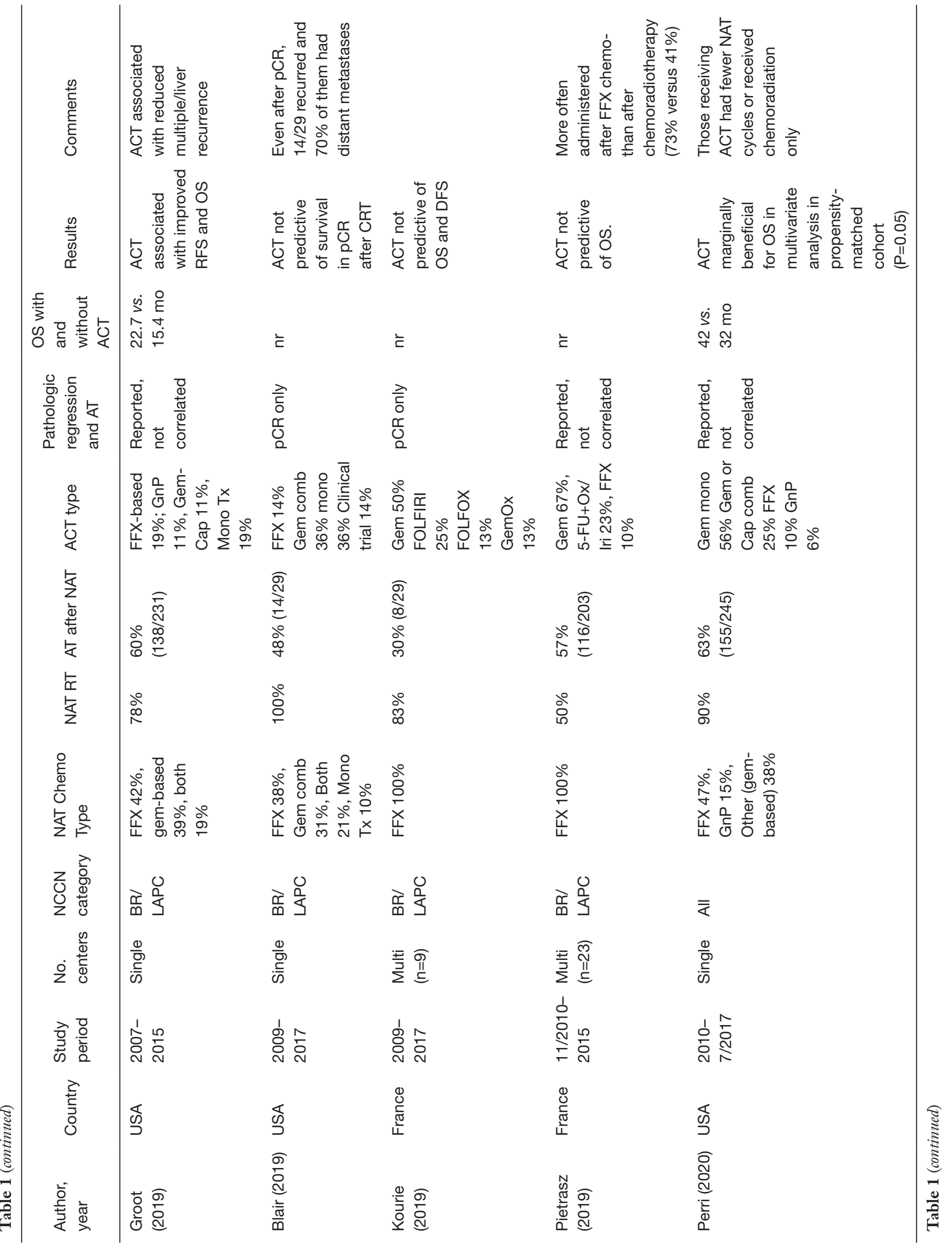




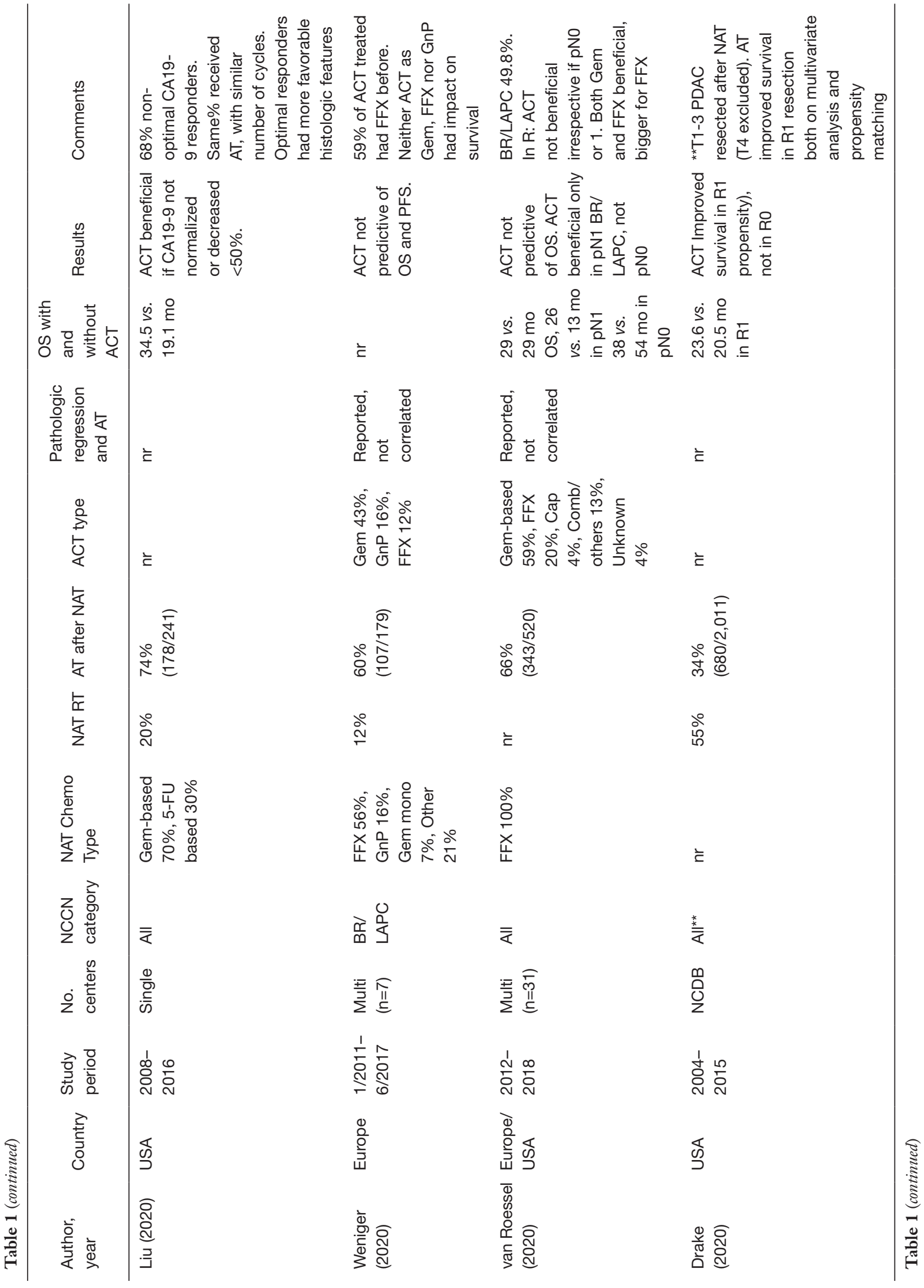

(C) Journal of Gastrointestinal Oncology. All rights reserved. $\quad$ f Gastrointest Oncol 2021;12(5):2521-2535 । https://dx.doi.org/10.21037/jgo-21-474 


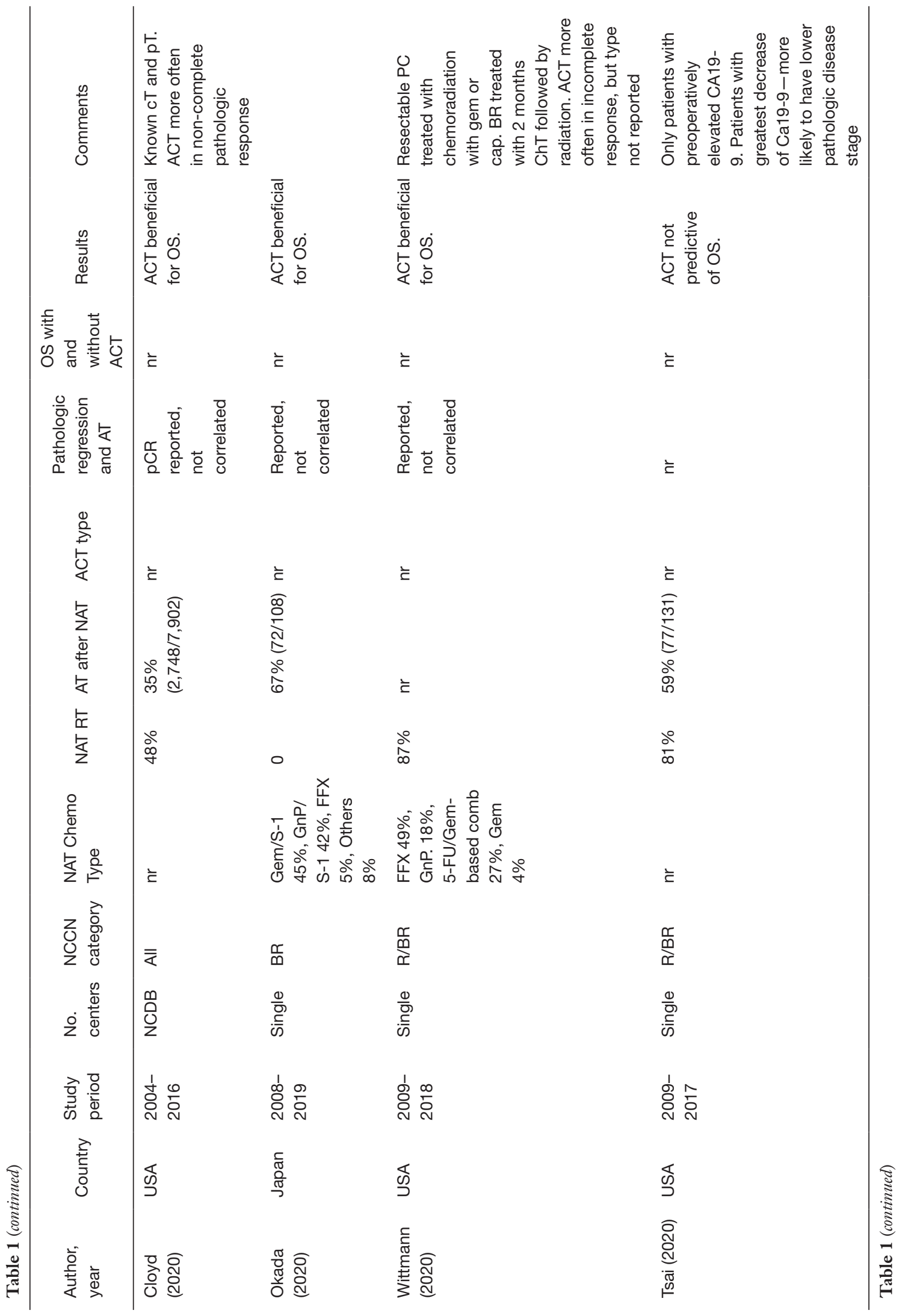

(C) Journal of Gastrointestinal Oncology. All rights reserved. $\quad 7$ Gastrointest Oncol 2021;12(5):2521-2535 I https://dx.doi.org/10.21037/jgo-21-474 


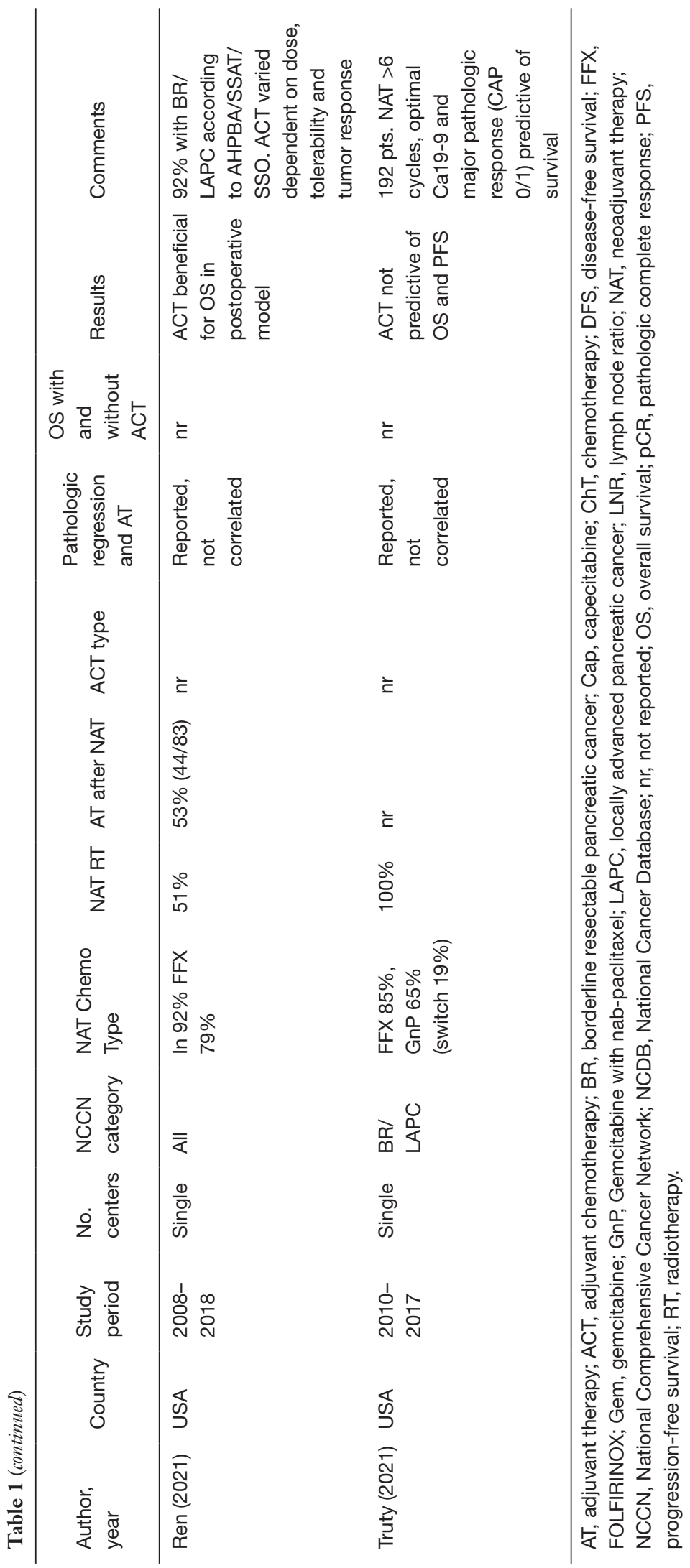

(C) Journal of Gastrointestinal Oncology. All rights reserved. $\quad$ J Gastrointest Oncol 2021;12(5):2521-2535 । https://dx.doi.org/10.21037/jgo-21-474 
2020. Many of the series are reports from single centers, including longer periods of time, when variating NAT regimen have been prevalent $(22,28-30)$. Others use data extracted from the National Cancer Database and therefore lack information on the particular type of NAT and ACT used (27,31-35). The studies reporting on the outcome in resected patients with $\mathrm{PC}$ receiving ACT after NAT are summarized in Table 1.

\section{Administration frequency and selection criteria for $A C T$}

The frequency with which ACT has been given varies among the studies between $14 \%$ and $75 \%$. In the dominating part, less than half of the patients have received treatment. Notably, in the large-scale reports based on the National Cancer Database, only about one third of the patients have received additional ACT after NAT and resection for PC (31-35). That is considerably less than the frequency with which ACT is administered after upfront resection reported repeatedly to be about $60 \%$ $(2,36)$. Barbas et al. found that ACT is particularly spared to the elderly, above 75 years of age, despite that they received NAT to the same extent as younger patients (37). The criteria for the application of ACT after NAT, beside tolerability, are seldom revealed, but few studies point out it is more often administered in patients with less favorable tumor characteristics on histology, such as regional lymph node metastases, R1 resection, and incomplete pathologic response $(27,35,38-40)$. Whether the reduced dose intensity or duration of NAT affects the decision to continue with ACT postoperatively has not been addressed.

\section{Survival outcome with ACT after NAT—matter of timing or total dose of chemotherapy}

The results whether ACT brings survival advantage after NAT in resected PC irrespective of resectability state preoperatively are contradictive. Eleven of the studies report no survival benefit $(13,21,29,32,41-47)$, while other fourteen find it beneficial $(22,28,30,31,33-35,38-40,48-51)$. The majority of the studies revealing advantage of ACT do not specify what the resectability status of the patients involved has been and only four of them $(22,28,38,48)$ provide data on the type of ACT administered. Interestingly, in half of the studies proving benefit of ACT, positive impact on survival has been observed not in the whole cohort, but only in specific subgroups of patients, such as having lymph node metastases, lower lymph-node ratio, R1 resection, and non- significant reduction of CA19-9 after NAT $(28,30,33,34,48)$.

Whether it is the exact timing (NAT, ACT or both) or it is the amount of chemotherapy delivered that has larger impact on survival is not quite clear. Epelboym et al. addressed particularly this issue in a single-institution series where the administered NAT and ACT were similargemcitabine-based in $73 \%$ and $85.5 \%$, respectively, and 5 -FU based in $21 \%$ and $8.3 \%$ (29). In this situation with the same NAT and ACT, the entire duration of the oncologic treatment was found to be more important than the timing itself. ACT of at least 6 cycles had similar benefit as combination of NAT and ACT with the same duration. On the contrary, van Roessel et al. reported that similar number of NAT cycles were administered to patients with and without ACT and still additional ACT had no benefit in the common cohort (48). The type of chemotherapy in NAT and ACT, though, was not specified and matched. They found, however, in a subgroup of patients with nodepositive BR/LAPC in which ACT was beneficial, that the effect modification was dependent of fewer number of NAT cycles. Dhir et al. investigated the value of ACT in patients who received NAT in form FOLFIRINOX or $\mathrm{GnP}$ and found that ACT was predictive of survival in one multivariate model, while in another model, the total number of NAT/ACT cycles to be associated with improved survival (50). The type of ACT administered was not reported. In a series of patients with only earlystage PC (IA-IB), Vega et al. found that any sequence of chemotherapy given (NAT, ACT or perioperative NAT/ ACT) lead to improved survival compared to if not chemotherapy was given at all (52). They did not provide data on the type of chemotherapy applied either (52). Thus, the question whether the total dose or the time-point of chemotherapy is more important, remains to be answered.

\section{Is there benefit of ACT after NAT in BR and LAPC?}

Only three of the eleven studies focusing specifically on $\mathrm{BR} / \mathrm{LAPC}$, and being the largest ones, revealed survival advantage of ACT $(22,48)$. Groot et al. reported the largest single-center series of BR/LAPC where 138 of 231 patients received ACT and also the only one showing ACT is advantageous both for the overall and recurrencefree survival. ACT was associated with reduced number of liver and multiple-site recurrence (22). van Roessel et al. found in a multi-institutional cohort that ACT, given to 159 of $259 \mathrm{BR} / \mathrm{LAPC}$ patients, was beneficial for survival only in lymph-node positive, but not in node-negative BR/ 
LAPC patients (48). Okada et al. who reported the only study on purely BRPC and the only study coming from the East, where S-1 was used as part of gemcitabine-based combination therapy in NAT in $87 \%$ of the patients (51). They found ACT to improve the survival of resected BR patients. Although the type of ACT was not specified, reference to previous publications reveals that the adjuvant regimen was probably similar-combination of gemcitabine/ S-1. The total number of cycles of chemotherapy in NAT/ ACT were not reported. The remaining smaller studies did not show any additional survival benefit of ACT, but might as well have been underpowered to detect a difference.

\section{ACT after FOLFIRINOX in BR/LAPC}

Four studies reported data where only FOLFIRINOX was used as NAT. In two of them, reporting multicenter data from France, ACT was dominated by gemcitabine in 67 and $77 \%$, respectively, while FOLFIRINOX was very seldom continued-in about $9 \%$ of patients $(41,45)$. ACT was not found to play a role for the overall survival. No subgroup analysis was performed to correlate pathologic response and the value of additional treatment. A third study, also from France, looked only at patients with complete pathologic response (pCR) after FOLFIRINOX. Only $30 \%$ of patients received ACT (gemcitabine in 50\% and no FOLFIRINOX) and it had no impact on survival (44). In the fourth already mentioned study by van Roessel et al., ACT was also dominated by gemcitabine-based treatment in 59\% while FOLFIRINOX was applied in $20 \%$ of cases, but it was not reported whether specifically the BR/LAPC group received ACT with the same proportion of drugs (48). Both gemcitabine and FOLFIRINOX showed advantage given as ACT, particularly the latter.

\section{ACT after other NAT in BR/LAPC}

The remaining studies used various NAT regimens as the proportion of FOLFIRINOX in them varied between 38\% and $85 \%$. In four them the ACT type was reported, mostly dominated by gemcitabine except for one $(13,22,41,43)$. In the only series selectively performed in LAPC, Gemenetzis et al. reported similar proportion of FOLFIRINOX to be given both as NAT and ACT, $63 \%$ and 64\%, yet, proving no advantage of ACT (13). It was not reported whether patients tended to be kept on the same NAT and ACT regimens or not. Radiotherapy was part of NAT in more than half of the patients and up to $100 \%$ in all the series, but whether its application would modify the efficacy of ACT has not been addressed.

How to interpret the data on ACT in BR/LAPC from the current reports is somehow ambiguous. The studies are relatively small to address this particular issue. The patients undergoing surgery after NAT are already a selected cohort that carries the common feature of response to particular regimen by showing stabilization or regression during treatment. To look further for effectiveness of various ACT, one should probably look into subgroups of patients stratified according to treatment response to NAT and the type of additional treatment, which would demand much larger cohorts. This information on the treatment sequence in each patient is lacking in the literature-whether the type of NAT and ACT administered was similar or not and whether the pathologic response to NAT have influenced the choice and efficiency of ACT. So, would it be fair to generalize in favor or not of ACT when so many important variables are not taken into consideration?

\section{To whom should ACT be given after NAT?}

Data from the focused BR/LAPC studies was inconclusive whether ACT might have a benefit for the whole cohort of patients or only for a certain subgroup. Therefore, we looked broader for further details in the remaining studies reporting ACT after NAT for all categories of resected PC (Table 1) in which BR and LAPC were at least part of the cohorts. Of the ten studies reporting definitive beneficial effect of ACT, in five advantage was observed only in a subgroup of the patients.

\section{ACT in the presence of lymph node metastases}

The most studied correlation is the efficacy of ACT in respect to the lymph node status after resection. Lymph node metastases and lymph node ratio (LNR) are negative prognostic factors for survival and can be referred to as signs of tumor aggressiveness and/or relative insensitivity to the administered NAT $(22,38)$. Respectively, additional potent chemotherapy might presumably be of benefit. ACT has more often been administered to N1 patients after NAT $(33,40,48)$. Roland et al. and Swords et al. reported that patients with lower $\mathrm{LNR}<0.15$ were the ones who benefit from additional ACT $(28,33)$. In the study by Roland et al. both NAT and ACT were similar gemcitabine/capecitabine-based (28). That could point out that whenever tumor aggressiveness is greater or the tumor 
is not responsive to the administered lower-potency NAT, as reflected by higher LNR, continuous treatment with similar chemotherapy might not be beneficial. In van Roessel's study, node-positive patients with BR/LAPC had benefit of ACT irrespective if it was gemcitabine-based or FOLFRINOX (48). Interestingly, the same study did not find ACT to improve survival in N1 primary resectable cancer, but details on the profile of the administered NAT and ACT in this case were not provided. Other studies did not find correlation between lymph node status and benefit of ACT (32).

\section{ACT and pathologic regression}

While it is more difficult to assume what the lymph node status has initially been before NAT, the pathologic regression of the primary tumor gives a more quantitative impression of the therapeutic response. It describes the proportions of post-treatment fibrosis and the remaining viable tumor and has been clearly associated with survival. Two studies looked at patients with pCR after NAT. Kourie et al. did not find additional benefit of ACT after FOLFIRINOX and pCR (44). None of the patients in the study received FOLFIRINOX as ACT. Blair et al. evaluated 29 patients with pCR all of whom received radiotherapy. Despite that about half of the patients developed recurrence after pCR $(n=14 / 29)$, and of them $70 \%$ had distant metastases, indicating that the systemic control of disease was failing, no benefit of ACT was seen (43). In this study, FOLFIRINOX represented only $38 \%$ of NAT and $14 \%$ of ACT, the latter being dominated by gemcitabine and monotherapy in $2 / 3$ of the cases. Thus, in case of pCR, whether FOLFIRINOX given as ACT would provide additional survival benefit, particularly if it has been the regimen in the induction chemotherapy, remains to be investigated. As for the most common scenario, when incomplete pathologic response is observed, there are no studies that correlated it with the efficacy of the administered ACT.

\section{ACT and biochemical response}

Biochemical response after NAT and correlation with the efficacy of ACT has also been investigated. Liu et al. found that ACT improved the overall survival of patients who did not have normalized serum CA19-9 or in whom it was reduced by less than $50 \%$ after gemcitabine and 5 -FU-based NAT (30). The type of ACT was not reported in the study.

\section{ACT and genetic profile of the tumor}

Finding a genetic signature that reveals sensitivity to chemotherapy is a long-tempted mean for personalized patient approach in order to fine-tune the treatment selection. The most widely addressed expression of hENT1 as a marker for sensitivity to gemcitabine, has been found to correlate with survival in patients with BR treated with gemcitabine-based neoadjuvant chemoradiotherapy (53). However, whether patients had further benefit of continuous ACT was not addressed in this study. Using the PC mutational profile could potentially identify responders to platinum (54) or other targetable drugs (55). Whether these correlated with the histologic response found post factum after NAT or whether targeted ACT to detected would have additional benefit has not been investigated.

\section{Which type of ACT is most efficient after NAT?}

Optimally, the clinical question would be whether the given NAT has sufficient effectiveness, as predicted by the observed pathologic and biochemical response, and whether treatment should continue postoperative with the same agent(s). Like addressed previously, there is no study that investigates this issue. van Roessel et al. are the only ones to address which type of ACT is more effective after single type of NAT (FOLFIRINOX), finding that both continuation of FOLFIRINOX as well as gemcitabinebased ACT was effective in N1 BR/LAPC (48). Further data was not presented on whether the same ACT or a therapy switch to gemcitabine may have had impact on survival depending on pathologic tumor response. Kourie et al. showed that in patients with pCR after FOLFIRINOX, gemcitabine and fewer-agents ACT do not further improve survival (44). No one in this series continued on FOLFIRINOX, so it is unclear whether further benefit could have been obtained from the same regimen.

The majority of the remaining studies report heterogeneous NAT and ACT, without correlation on what premise the choice of ACT has been made. Therefore, hardly any conclusions can be drawn on what the optimal choice of ACT should be. In the two studies where gemcitabine or 5-FU-based treatment was used both in the pre- and the postoperative setting, ACT seems worthwhile in case of more favorable histology-e.g., either if the tumor does not express very aggressive features (higher LNR) or whenever at least some tumor response to chemotherapy is present (28). The same might be true 
even for FOLFIRINOX, as suggested by van Roessel et al. Further studies are needed to assess whether greater number of cycles bring additional benefit in responders and whether gemcitabine may still prove efficient in case tumor is fairly insensitive to FOLFIRINOX.

In summary, data on whether and what type of ACT is beneficial after NAT and resection for BR/LAPC are very limited and heterogeneous. Larger studies suggest that ACT may bring survival advantage and should be attempted particularly in node-positive disease and preferably with more potent regimen such as FOLFIRINOX, if tolerable. In case of complete pathologic response, particularly after FOLFIRINOX, it does not seem beneficial to deescalate the treatment during ACT, but whether continuation on the same regimen is worthwhile needs to be further examined. In case of gemcitabine-based treatment as NAT, continuation with more cycles seems to be of value unless tumor biology proves to be too aggressive, with high LNR. Whether switch to a different regimen should be sought, if tolerability allows it, needs to be further studied. Whether it is the exact timing of administration or the total dose of chemotherapy that impacts survival, especially for FOLFIRINOX and gemcitabine-Nab-paclitaxel, is unknown. Generally, there is a strong and calling need for further research assessing patient-level data on response to oncologic therapy and using this information to personalize ACT for the most effective postoperative treatment.

\section{Acknowledgments}

Funding: None.

\section{Footnote}

Provenance and Peer Review: This article was commissioned by the editorial office, Fournal of Gastrointestinal Oncology for the series "Surgery for Locally Advanced Pancreatic Cancer". The article has undergone external peer review.

Conflicts of Interest: Both authors have completed the ICMJE uniform disclosure form (available at https:// dx.doi.org/10.21037/jgo-21-474). The series "Surgery for Locally Advanced Pancreatic Cancer" was commissioned by the editorial office without any funding or sponsorship. Elena Rangelova served as the unpaid Guest Editor of the series. Elena Rangelova reports that she receives honoraria by ESGAR for invited expert lectures during workshops, unrelated to current series. The authors have no other conflicts of interest to declare.

Ethical Statement: The authors are accountable for all aspects of the work in ensuring that questions related to the accuracy or integrity of any part of the work are appropriately investigated and resolved.

Open Access Statement: This is an Open Access article distributed in accordance with the Creative Commons Attribution-NonCommercial-NoDerivs 4.0 International License (CC BY-NC-ND 4.0), which permits the noncommercial replication and distribution of the article with the strict proviso that no changes or edits are made and the original work is properly cited (including links to both the formal publication through the relevant DOI and the license). See: https://creativecommons.org/licenses/by-nc-nd/4.0/.

\section{References}

1. Wagner M, Redaelli C, Lietz M, et al. Curative resection is the single most important factor determining outcome in patients with pancreatic adenocarcinoma. Br J Surg 2004;91:586-94.

2. Socialstyrelsen. Cancer i siffror: populärvetenskapliga fakta om cancer. 2018.

3. Groot VP, Gemenetzis G, Blair AB, et al. Defining and Predicting Early Recurrence in 957 Patients With Resected Pancreatic Ductal Adenocarcinoma. Ann Surg 2019;269:1154-62.

4. Neoptolemos JP, Moore MJ, Cox TF, et al. Effect of adjuvant chemotherapy with fluorouracil plus folinic acid or gemcitabine vs observation on survival in patients with resected periampullary adenocarcinoma: the ESPAC-3 periampullary cancer randomized trial. JAMA 2012;308:147-56.

5. Neoptolemos JP, Stocken DD, Friess H, et al. A randomized trial of chemoradiotherapy and chemotherapy after resection of pancreatic cancer. $\mathrm{N}$ Engl J Med 2004;350:1200-10.

6. Neoptolemos JP, Palmer DH, Ghaneh P, et al. Comparison of adjuvant gemcitabine and capecitabine with gemcitabine monotherapy in patients with resected pancreatic cancer (ESPAC-4): a multicentre, open-label, randomised, phase 3 trial. Lancet 2017;389:1011-24.

7. Uesaka K, Boku N, Fukutomi A, et al. Adjuvant chemotherapy of S-1 versus gemcitabine for resected pancreatic cancer: a phase 3, open-label, randomised, noninferiority trial (JASPAC 01). Lancet 2016;388:248-57. 
8. Bockhorn M, Uzunoglu FG, Adham M, et al. Borderline resectable pancreatic cancer: a consensus statement by the International Study Group of Pancreatic Surgery (ISGPS). Surgery 2014;155:977-88.

9. Conroy T, Desseigne F, Ychou M, et al. FOLFIRINOX versus gemcitabine for metastatic pancreatic cancer. $\mathrm{N}$ Engl J Med 2011;364:1817-25.

10. Von Hoff DD, Ervin T, Arena FP, et al. Increased survival in pancreatic cancer with nab-paclitaxel plus gemcitabine. N Engl J Med 2013;369:1691-703.

11. Hackert T, Sachsenmaier M, Hinz U, et al. Locally Advanced Pancreatic Cancer: Neoadjuvant Therapy With Folfirinox Results in Resectability in $60 \%$ of the Patients. Ann Surg 2016;264:457-63.

12. Michelakos T, Pergolini I, Castillo CF, et al. Predictors of Resectability and Survival in Patients With Borderline and Locally Advanced Pancreatic Cancer who Underwent Neoadjuvant Treatment With FOLFIRINOX. Ann Surg 2019;269:733-40.

13. Gemenetzis G, Groot VP, Blair AB, et al. Survival in Locally Advanced Pancreatic Cancer After Neoadjuvant Therapy and Surgical Resection. Ann Surg 2019;270:340-7.

14. Rangelova E, Wefer A, Persson S, et al. Surgery Improves Survival After Neoadjuvant Therapy for Borderline and Locally Advanced Pancreatic Cancer: A Single Institution Experience. Ann Surg 2021;273:579-86.

15. Labori KJ, Lassen K, Hoem D, et al. Neoadjuvant chemotherapy versus surgery first for resectable pancreatic cancer (Norwegian Pancreatic Cancer Trial - 1 (NorPACT-1)) - study protocol for a national multicentre randomized controlled trial. BMC Surg 2017;17:94.

16. Janssen QP, van Dam JL, Bonsing BA, et al. Total neoadjuvant FOLFIRINOX versus neoadjuvant gemcitabine-based chemoradiotherapy and adjuvant gemcitabine for resectable and borderline resectable pancreatic cancer (PREOPANC-2 trial): study protocol for a nationwide multicenter randomized controlled trial. BMC Cancer 2021;21:300.

17. Schorn S, Demir IE, Samm N, et al. Meta-analysis of the impact of neoadjuvant therapy on patterns of recurrence in pancreatic ductal adenocarcinoma. BJS Open 2018;2:52-61.

18. Papavasiliou P, Hoffman JP, Cohen SJ, et al. Impact of preoperative therapy on patterns of recurrence in pancreatic cancer. HPB (Oxford) 2014;16:34-9.

19. Conroy T, Hammel P, Hebbar M, et al. FOLFIRINOX or Gemcitabine as Adjuvant Therapy for Pancreatic Cancer. N Engl J Med 2018;379:2395-406.
20. Klaiber U, Schnaidt ES, Hinz U, et al. Prognostic Factors of Survival After Neoadjuvant Treatment and Resection for Initially Unresectable Pancreatic Cancer. Ann Surg 2021;273:154-62.

21. Truty MJ, Kendrick ML, Nagorney DM, et al. Factors Predicting Response, Perioperative Outcomes, and Survival Following Total Neoadjuvant Therapy for Borderline/Locally Advanced Pancreatic Cancer. Ann Surg 2021;273:341-9.

22. Groot VP, Blair AB, Gemenetzis G, et al. Recurrence after neoadjuvant therapy and resection of borderline resectable and locally advanced pancreatic cancer. Eur J Surg Oncol 2019;45:1674-83.

23. He J, Blair AB, Groot VP, et al. Is a Pathological Complete Response Following Neoadjuvant Chemoradiation Associated With Prolonged Survival in Patients With Pancreatic Cancer? Ann Surg 2018;268:1-8.

24. Chatterjee D, Katz MH, Rashid A, et al. Histologic grading of the extent of residual carcinoma following neoadjuvant chemoradiation in pancreatic ductal adenocarcinoma: a predictor for patient outcome. Cancer 2012;118:3182-90.

25. Chun YS, Cooper HS, Cohen SJ, et al. Significance of pathologic response to preoperative therapy in pancreatic cancer. Ann Surg Oncol 2011;18:3601-7.

26. Macedo FI, Ryon E, Maithel SK, et al. Survival Outcomes Associated With Clinical and Pathological Response Following Neoadjuvant FOLFIRINOX or Gemcitabine/ Nab-Paclitaxel Chemotherapy in Resected Pancreatic Cancer. Ann Surg 2019;270:400-13.

27. Sell NM, Lee GC, Fernández-Del Castillo C, et al. Evaluation of Pathologic Response on Overall Survival After Neoadjuvant Therapy in Pancreatic Ductal Adenocarcinoma. Pancreas 2020;49:897-903.

28. Roland CL, Katz MH, Tzeng CW, et al. The Addition of Postoperative Chemotherapy is Associated with Improved Survival in Patients with Pancreatic Cancer Treated with Preoperative Therapy. Ann Surg Oncol 2015;22 Suppl 3:S1221-8.

29. Epelboym I, Zenati MS, Hamad A, et al. Analysis of Perioperative Chemotherapy in Resected Pancreatic Cancer: Identifying the Number and Sequence of Chemotherapy Cycles Needed to Optimize Survival. Ann Surg Oncol 2017;24:2744-51.

30. Liu H, Zenati MS, Rieser CJ, et al. CA19-9 Change During Neoadjuvant Therapy May Guide the Need for Additional Adjuvant Therapy Following Resected Pancreatic Cancer. Ann Surg Oncol 2020;27:3950-60. 
31. Lutfi W, Talamonti MS, Kantor O, et al. Perioperative chemotherapy is associated with a survival advantage in early stage adenocarcinoma of the pancreatic head. Surgery 2016;160:714-24.

32. de Geus SWL, Kasumova GG, Eskander MF, et al. Is Neoadjuvant Therapy Sufficient in Resected Pancreatic Cancer Patients? A National Study. J Gastrointest Surg 2018;22:214-25.

33. Swords DS, Francis SR, Lloyd S, et al. Lymph Node Ratio in Pancreatic Adenocarcinoma After Preoperative Chemotherapy vs. Preoperative Chemoradiation and Its Utility in Decisions About Postoperative Chemotherapy. J Gastrointest Surg 2019;23:1401-13.

34. Drake JA, Stiles ZE, Behrman SW, et al. The utilization and impact of adjuvant therapy following neoadjuvant therapy and resection of pancreatic adenocarcinoma: does more really matter? HPB (Oxford) 2020;22:1530-41.

35. Cloyd JM, Ejaz A, Shen C, et al. Pathologic complete response following neoadjuvant therapy for pancreatic ductal adenocarcinoma: defining the incidence, predictors, and outcomes. HPB (Oxford) 2020;22:1569-76.

36. Skau Rasmussen L, Vittrup B, Ladekarl M, et al. The effect of postoperative gemcitabine on overall survival in patients with resected pancreatic cancer: A nationwide populationbased Danish register study. Acta Oncol 2019;58:864-71.

37. Barbas AS, Turley RS, Ceppa EP, et al. Comparison of outcomes and the use of multimodality therapy in young and elderly people undergoing surgical resection of pancreatic cancer. J Am Geriatr Soc 2012;60:344-50.

38. Perri G, Prakash L, Qiao W, et al. Postoperative Chemotherapy Benefits Patients Who Received Preoperative Therapy and Pancreatectomy for Pancreatic Adenocarcinoma. Ann Surg 2020;271:996-1002.

39. Wittmann D, Hall WA, Christians KK, et al. Impact of Neoadjuvant Chemoradiation on Pathologic Response in Patients With Localized Pancreatic Cancer. Front Oncol 2020;10:460.

40. Ren W, Xourafas D, Ashley SW, et al. Temporal Assessment of Prognostic Factors in Patients With Pancreatic Ductal Adenocarcinoma Undergoing Neoadjuvant Treatment and Resection. J Surg Res 2021;257:605-15.

41. Pietrasz D, Marthey L, Wagner M, et al. Pathologic Major Response After FOLFIRINOX is Prognostic for Patients Secondary Resected for Borderline or Locally Advanced Pancreatic Adenocarcinoma: An AGEO-FRENCH, Prospective, Multicentric Cohort. Ann Surg Oncol 2015;22 Suppl 3:S1196-205.

42. Chapman BC, Gleisner A, Rigg D, et al. Perioperative and Survival Outcomes Following Neoadjuvant FOLFIRINOX versus Gemcitabine Abraxane in Patients with Pancreatic Adenocarcinoma. JOP 2018;19:75-85.

43. Blair AB, Yin LD, $\mathrm{Pu}$, et al. Recurrence in Patients Achieving Pathological Complete Response After Neoadjuvant Treatment for Advanced Pancreatic Cancer. Ann Surg 2021;274:162-9.

44. Kourie H, Auclin E, Cunha AS, et al. Characteristic and outcomes of patients with pathologic complete response after preoperative treatment in borderline and locally advanced pancreatic adenocarcinoma: An AGEO multicentric retrospective cohort. Clin Res Hepatol Gastroenterol 2019;43:663-8.

45. Pietrasz D, Turrini O, Vendrely V, et al. How Does Chemoradiotherapy Following Induction FOLFIRINOX Improve the Results in Resected Borderline or Locally Advanced Pancreatic Adenocarcinoma? An AGEOFRENCH Multicentric Cohort. Ann Surg Oncol 2019;26:109-17.

46. Weniger M, Moir J, Damm M, et al. Respect - A multicenter retrospective study on preoperative chemotherapy in locally advanced and borderline resectable pancreatic cancer. Pancreatology 2020;20:1131-8.

47. Tsai S, George B, Wittmann D, et al. Importance of Normalization of CA19-9 Levels Following Neoadjuvant Therapy in Patients With Localized Pancreatic Cancer. Ann Surg 2020;271:740-7.

48. van Roessel S, van Veldhuisen E, Klompmaker S, et al. Evaluation of Adjuvant Chemotherapy in Patients With Resected Pancreatic Cancer After Neoadjuvant FOLFIRINOX Treatment. JAMA Oncol 2020;6:1733-40.

49. Franko J, Hsu HW, Thirunavukarasu P, et al. Chemotherapy and radiation components of neoadjuvant treatment of pancreatic head adenocarcinoma: Impact on perioperative mortality and long-term survival. Eur J Surg Oncol 2017;43:351-7.

50. Dhir M, Zenati MS, Hamad A, et al. FOLFIRINOX Versus Gemcitabine/Nab-Paclitaxel for Neoadjuvant Treatment of Resectable and Borderline Resectable Pancreatic Head Adenocarcinoma. Ann Surg Oncol 2018;25:1896-903.

51. Okada K, Murakami Y, Uemura K, et al. Neoadjuvant therapy for pancreatic cancer: an intention-to-treat analysis. Langenbecks Arch Surg 2020;405:623-33.

52. Vega EA, Kutlu OC, Salehi O, et al. Preoperative Chemotherapy for Pancreatic Cancer Improves Survival and R0 Rate Even in Early Stage I. J Gastrointest Surg 
2020;24:2409-15.

53. Yabushita Y, Mori R, Taniguchi K, et al. Combined Analyses of hENT1, TS, and DPD Predict Outcomes of Borderline-resectable Pancreatic Cancer. Anticancer Res 2017;37:2465-76.

54. Waddell N, Pajic M, Patch AM, et al. Whole genomes redefine the mutational landscape of pancreatic cancer.

Cite this article as: Rangelova E, Bratlie SO. How to select the most appropriate adjuvant treatment after neoadjuvant treatment and resection for locally advanced pancreatic cancer? J Gastrointest Oncol 2021;12(5):2521-2535. doi: 10.21037/jgo-21474
Nature 2015;518:495-501.

55. Singhi AD, George B, Greenbowe JR, et al. Real-Time Targeted Genome Profile Analysis of Pancreatic Ductal Adenocarcinomas Identifies Genetic Alterations That Might Be Targeted With Existing Drugs or Used as Biomarkers. Gastroenterology 2019;156:2242-2253.e4. 